\title{
The irritable colon syndrome
}

\author{
A. M. CONNEll \\ Department of Surgery, \\ Queen's University of Belfast
}

ThE IRRITABLe colon is a symptom complex rather than a pathological entity and may be merely one manifestation of a more generalized muscular disorder of the gastro-intestinal tract. The syndrome masquerades under a variety of names such as spastic colon, irritable bowel, mucous colitis, functional bowel disorder, etc., but possibly 'irritable colon' is the most valid description.

The condition is very common although a firm diagnosis is made less frequently than it ought, possibly on account of the absence of definitive pathological criteria. Some gastroenterologists regard it as the most common gastro-intestinal complaint and it is a source of considerable morbidity in the community. While most sufferers can live tolerably comfortably, there is a minority which in absolute terms represents a large number of persons, in whom symptoms result in a serious disruption of social and business life. The social consequences are greatly magnified in areas where dysentery is endemic and a postdysenteric irritable bowel is an extremely common chronic complaint.

\section{Physical syndromes}

Two distinct conditions have to be distinguished (Chaudhary \& Truelove, 1961a). The first is illustrated by the patient who has persistent looseness of the bowels, usually in the morning, without pain or any other significant symptoms. The bowel frequency seems to mirror life-stress and this condition is best described as nervous diarrhoea. It should be regarded as distinct from the irritable colon syndrome in all aspects including management and therapy.

The diagnosis of irritable colon is one of exclusion and to make it, at least three conditions must be satisfied. There must be a history of disturbance of bowel habit, either constant or intermittent, abdominal pain, usually related to the bowel dysfunction, and an absence of any radiological, bacteriological, pathological or biochemical abnormality. The pain need not be confined to the left iliac fossa, and central abdominal pain is common. Pain aggravated by eating or affected by defaecation or the passage of flatus is suggestive. The change in bowel habit may be towards constipation, diarrhoea or complete irregularity. The passage of small hard scyballous stools (rabbit stools) is common. Typically, the sufferer remains well for several weeks when he suddenly develops debilitating attacks accompanied by profuse diarrhoea and abdominal pain lasting 24-48 hr. Abdominal distension, flatulence, heartburn and nausea are frequent accompaniments.

\section{Aetiological considerations}

The aetiology of the condition is unknown, but study of the clinical histories uncovers at leas five factors.

\section{(a) Post-dysenteric}

Many patients, particularly in warm climates, date their symptoms to a previous attack of para sitic infestation of the alimentary tract, ofter amoebic dysentery. Considering the world-wide incidence, post-dysenteric irritable bowel is one of the most common of all clinical conditions and in Great Britain accounts for some $25 \%$ of all cases of irritable colon (Chaudhary \& Truelove, 1961a). In these cases vegetative forms of parasites are not seen. It may be that small foci of encysted parasites persist resulting in neuro-muscular irritability or else the previous active infection results in a prolonged functional disorganization of the muscle of this bowel.

\section{(b) Constitutional}

In temperate climates patients may recollect symptoms persisting for many years and sometimes parents disclose that their child was 'colicky' so it may be that some persons have an inborn irritability of intestinal smooth muscle.

\section{(c) Post-vagotomy}

One clue which cannot be overlooked is that a proportion of persons who have had vagotomy for peptic ulceration develop symptoms indistinguishable from those of the irritable colon syndrome. Indeed, the term 'post-vagotomy irritable colon' is a more accurate description than 'post-vagotomy diarrhoea'. 


\section{(d) Emotional}

The role of emotion is always quoted in these cases. Not infrequently these patients have some disturbance of behaviour or attitudes, but a great many have no more than is expected in a normal member of the community. To call this condition psychosomatic and feel that this has solved the problem of aetiology is both facile and unhelpful. It is likely that there is a genuine clinical basis for the disturbance of function but where life stress intrudes the symptoms become less tolerable or else form the basis for a hysterical reaction to the stress.

\section{(e) Secondary to other conditions}

In many cases the picture is complex and symptoms of irritable colon exist in association with known pathology elsewhere, such as duodenal ulcer, gall bladder disease, post-operative states, or a gynaecological lesion.

\section{Mechanisms}

\section{Pressure studies}

Studies of the alimentary pressures have revealed something of the functional defect in the irritable colon. Pressure recordings taken at a time when the patient is in remission show no deviation from normal, but when studies are done during a period of symptoms an exaggerated degree of altered pressure activity can be detected (Chaudhary \& Truelove, 1961b ; Connell, Avery Jones \& Rowlands, 1965; Wangel \& Deller, 1965). This is demonstrated in two waysin the frequency of contraction of the colon, and in the overall muscular work done by it.

Muscular activity can be detected in the normal colon for up to $50 \%$ of the time of recording and the activity at any one area often occurs in bursts alternating with periods of relative quiescence. Where activity is present, pressure waves occur at a frequency of approximately 2 per minute and only rarely are faster rhythms detected. However, in the irritable colon syndrome faster rhythms are seen more frequently than normal (Parks, Connell \& Misiewicz, 1968) and this difference is exaggerated by a cholinergic stimulus such as prostigmine. The total amount of activity is also greater than normal after stimuli such as prostigmine both in the irritable colon of temperate climates (Chaudhary \& Truelove, 1961 b) and in post-dysenteric irritable colon (Connell et al., 1964).

Even more germane to the problem than the response to prostigmine is the response to a repetitive physiological stimulus such as eating. In the normal subject the ingestion of food results in an increase in the motility of the sigmoid of approximately $50 \%$. However, in a proportion of patients with irritable colon in whom eating causes exaggeration of symptoms, pressure recordings taken before and after food at a time when symptoms are present show a grossly exaggerated response to eating (Connell et al., 1965).

It is not possible to define the gastrocolic responses in terms of nervous pathways and it has been postulated that they have a humoral origin (Logan \& Connell, 1967). Recent studies involving cross-circulation or auto-transfusion experiments in cats or dogs have indicated that this is almost certainly so (Logan, 1966; Torsoli, 1968). Gastrin is a possible humoral mediator and both gastrin II and pentagastrin alter the motility of the sigmoid colon (Logan \& Connell, 1967). Intravenous infusion of pentagastrin in physiological doses results in an increase in sigmoid motility of the same order as occurs after eating although this has not been established with mathematical significance (Misiewicz, Holdstock \& Waller, 1967). In vitro, gastrin II added to an organ bath stimulated smooth muscle isolated from the human sigmoid colon in seven of thirteen experiments (Bennett, Misiewicz \& Waller, 1967). Further investigation of these responses may provide further clues to the problem of the irritable colon.

\section{The significance of pressure waves}

Pressure waves by themselves are not a cause of pain but are best regarded as demonstrating the existence of pressure segments in the alimentary tract and loosely as an index of the resistance to transit along the gut (Connell, 1965; Painter et al., 1965). Increased pressure activity represents sigmoid resistance and a tendency to delay transit of faeces or flatus. This may cause distension proximally in the colon, particularly if at the same time there is a caudal pressure gradient resulting from the emptying of the ileum into the colon which also occurs after eating (MacEwan, 1904). It is this distension which is the cause of the symptoms (Fig. 1).

\section{Radiology}

These pressure changes are the manometric analogue of the radiological appearances frequently seen in irritable colon, where there may be a contracted, irregular colon (Fig. 2). This is not a specific appearance but it is more likely to be seen in a patient with irritable colon than in a normal patient, just as there is a statistical probability that manometric overactivity may be detected in these patients. Thus, patients with irritable colon appear to have an overshoot of a normal response which could be due either to an 
unduly powerful drive to the motor activity of the colon or to the suppression of normal feedback mechanisms.

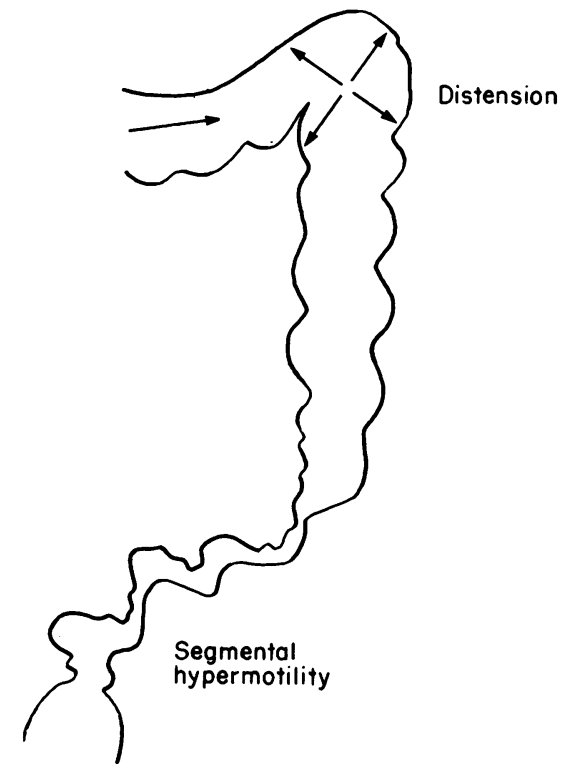

FIG. 1. Diagrammatic representation of the mechanism whereby symptoms may be produced in the irritable colon syndrome. [From Connell, A.M. (1968) Handbook of Physiology (Ed. by C.F. Code and W. Heidel). Williams \& Watkins, Baltimore. By kind permission of the publishers.]

\section{Diagnosis}

Any diagnosis of exclusion is open to pitfalls and the absolute exclusion of serious conditions is difficult. Carcinoma of the alimentary tract may present with symptoms indistinguishable from irritable colon. A small area of Crohn's disease may be very difficult to detect and signals its presence only by the occurrence of symptoms of irritable colon. A common pitfall exists in the diagnosis of lesions about the pylorus, especially antral carcinoma which, not infrequently, present with disorders of bowel habit and abdominal pain with masking of upper gastro-intestinal signs or symptoms.

It is unrealistic, however, to subject all patients, especially young females, with symptoms of irritable colon to a rigorous gastro-intestinal investigation. In history taking and examination attention must be paid to weight-loss. Patients with primary irritable colon do not normally lose weight. Symptoms and signs of degenerative changes in the circulatory system suggest an ischaemic colitis. Eye signs, skin rashes and joint pains suggest ulcerative colitis, Crohn's disease,

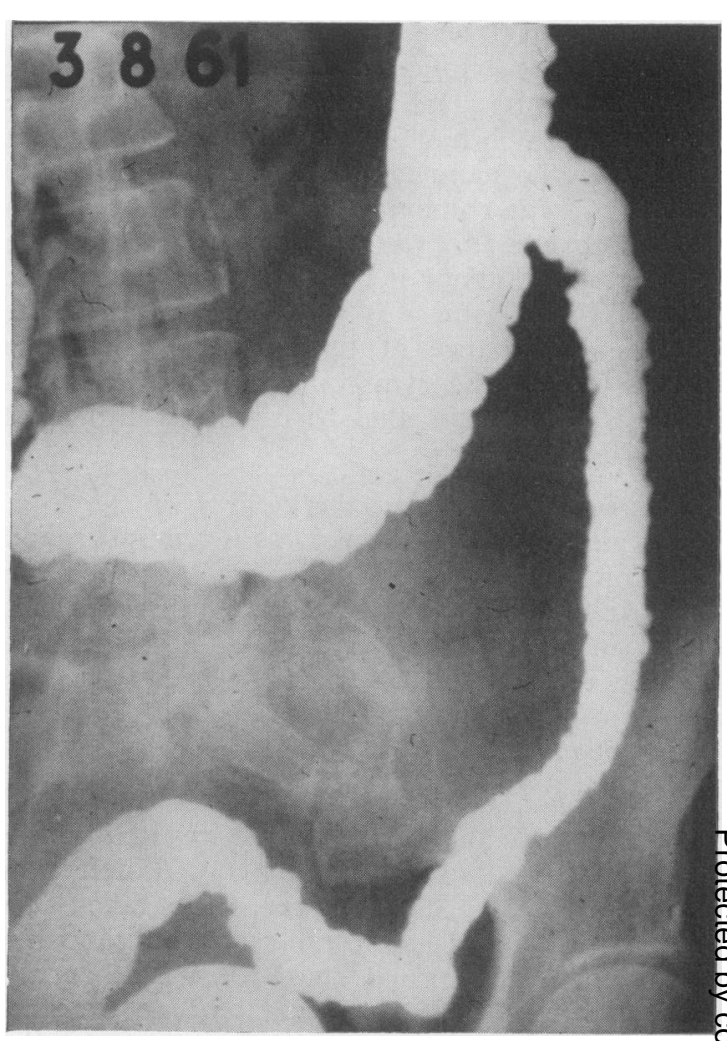

FIG. 2. Radiological appearances in a patient with irritable colon syndrome.

or a small intestinal lesion. Simple investigations which are obligatory and exclude many organic causes are examination of the stool for occult blood, estimation of haemoglobin, white count and ESR, examination of the stool for pathogens, and a rectal examination. Sigmoidoscopy should be part of the routine clinical examination of all patients with bowel symptoms. If any of these are abnormal, the condition is something other than primary irritable colon. In younger patients this, together with follow-up, is probably adequate, but in older patients a gastro-intestinal barium series is usually indicated.

\section{Treatment}

The condition persists for many years and the main element of treatment is to support patients through periods of relapse. This will involve drug therapy less than attention to the control of acute and chronic stresses in life situations. Where positive indications for sedation exist it should not be withheld, but as a long-term therapy it is severely limited. 
Symptomatic treatment can be helpful. Bulking agents and anti-diarrhoeal agents such as codeine phosphate or diphenoxylate have their place. Standardized senna given in sublaxative doses can be helpful in decreasing bowel resistance and relieving distension. Antispasmodics can be useful although belladonna alkaloids have a paradoxical effect on bowel motility and, when tested experimentally, therapeutic doses may result in the fast wave pattern characteristic of irritable colon. This may, in part, explain their disappointing effect in low dosage, although at higher dosage they suppress the motor activity of the sigmoid. The musculotropic agent mebeverine diminishes bowel activity both under basal conditions and after stimulation by prostigmine. A double blind clinical trial has indicated that patients treated with mebeverine feel better than a control group given a placebo (Connell, 1964).

\section{Conclusion}

Because in the past irritable colon has been firmly labelled functional, it has failed to attract the attention deserved by a condition producing such widespread morbidity in the community. Now that an indication exists of the mechanisms whereby the symptoms of irritable colon may develop it should be possible to place clinical management on a firmer, more rational basis.

\section{References}

Bennett, A., Misiewicz, J.J. \& Waller, S.L. (1967) Analysis of the motor effects of gastrin and pentagastrin on the human alimentary tract. Gut, 8, 470 .

Chaudhary, N.A. \& Truelove, S.C. (1961a) The irritable colon syndrome. Quart. J. Med. 31, 307.
Chaudhary, N.A. \& Truelove, S.C. (1961b) Human colonic motility: a comparative study of normal subjects, patients with ulcerative colitis and patients with the irritable colon syndrome. Gastroenterology, 40, 1.

Connell, A.M. (1964) Physiological and clinical assessment of the effect of the musculotropic agent mebeverine on the human colon. Brit. med. J. ii, 848.

ConNELl, A.M. (1965) The significance of pressure waves in the sigmoid colon. Amer. J. dig. Dis. 10, 455.

Connell, A.M., Avery Jones, F. \& Rowlands, E.N. (1965) The motility of the pelvic colon. IV. Abdominal pain associated with colonic hypermotility after meals. Gut, 6, 105.

Connell, A.M., Hassanein, M.A., GaAfer, M. \& Khayal, M.A. (1964) Motility of the pelvic colon. III. Motility responses in patients with symptoms following amoebic dysentery. Gut, 5, 443.

LOGAN, C.J.H. (1966) The role of gastrin in the control of intestinal motility. M.S. Thesis, Queen's University, Belfast.

Logan, C.J.H. \& ConNell, A.M. (1967) The role of gastrin in gastro-ileo-colic responses. Amer. J. dig. Dis. 12, 277.

Misiewicz, J.J., Holdstock, D.J. \& Waller, S.L. (1967) Motor responses of the human alimentary tract to near maximal infusions of pentagastrin. Gut, 8, 463.

MacEwan, W. (1904) The function of the caecum and appendix. Lancet, ii, 995.

Painter, N.S., Truelove, S.C., Ardran, G.M. \& Tuckey, M. (1965) Segmentation and the localization of intraluminal pressures in the human colon with special reference to the pathogenesis of colonic diverticula. Gastroenterology, 49, 169.

Parks, T.G., Connell, A.M. \& Misiewicz, J.J. (1968) Studies in diverticular disease. III. A comparison with the irritable bowel syndrome using miniature balloon technique. (In preparation).

Torsoli, A., Ramorino, M.L. \& Crucioli, V. (1968) Proceedings of the International Motility Symposium, Belfast, 1967. Amer. J. dig. Dis. 13, 462.

Wangel, A.G. \& DelleR, D.J. (1965) Intestinal motility in man. III. Mechanisms of constipation and diarrhoea with particular reference to the irritable colon syndrome. Gastroenterology, 48, 69. 